\title{
STUDY OF ALGAL DIVERSITY OF DAM TAL-AKOLE, DIST- AHMEDNAGER, MAHARASHTRA
}

\author{
Deepmala B. Tambe* and Balasaheb K. Tapale \\ Adv. M. N. Deshmukh Arts, Com. and Science College Rajur, Tal-Akole Dist-A. Nagar \\ Corresponding Author: tambedeepmala@gmail.com
}

Communicated : 22.2.20

Revision :15.03.20 \& 20.4.2020

Accepted : 22.05.2020

Published: 30.05 .2020

\begin{abstract}
Ambit Dam is located at Ambit village in western part of the Ahmednagar district in Maharashtra. It build across the river Mula near the village Ambit. During the present study qualitative analysis of algal diversity of Ambit dam has been carried out in one year (June 2018-May 2019). Three sampling sites were selected for collection of water samples. In monsoon season the flow of water was quiet fast later on water become steady and stagnant. Many planktonic, benthic and epiphytic algae were enumerate during investigation period from two sampling sites. The algae were identified with the use of standard monograph. The systematic account of the algal taxa were made for four groups i.e. Cyanophyceae, Bacillariophyceae, Chlorophyceae and, Xanthophyceae and. In general there is good flora in Ambit dam.
\end{abstract}

Keywords: Diversity, Ambit Dam, Algal diversity

\section{INTRODUCTION:}

Ambit Dam is located at Ambit village in western part of the Ahmednagar district in Maharashtra. The Ahmednagar district is located between $18{ }^{\circ} 26$ and 19०59 North latitude and 73040 and $75 \circ 43$ East longitude. Ambit dam is built across the river Mula, near the village Ambit. This dam is build up with RCC technology. Height of dam above lowest foundation is 24 meter while length is 242 meter. The live storage is of water is 195 MCFT. The main sources of water for dam are streams, rivers, fountains etc. The annual rainfall up to Ghatghar is $5460 \mathrm{~mm}$. and on dam $3225 \mathrm{~mm}$. The total irrigated area under the dam is 68,63 $\mathrm{Km}^{2}$.

\section{MATERIALS AND METHODS}

Three sampling stations of Ambit dam were selected for the collection of algal samples during the one-year study.

Station-S1. Station-S2. Station - S3.
The water samples were collected monthly from the selected three stations over a period of one year June 2018 to May 2019.The monthly collection of the sample was in morning between 9 to $11 \mathrm{am}$. Throughout the investigation. The samples were collected with plankton net 25 meshes bolting silk of diameter of pore 60 micro meters (Trivedi and Goel, 1986). The samples were studied qualitatively. The collected algal samples were observed freshly and also preserved in $4 \%$ formaldehyde or Lugols iodine solution. Phytoplanktons were observed in fresh living condition as well as after boiling in nitric acid (Sinha 1997). They were micro photographed. The systematic accounts of the algal taxa were made for four groups as follow.
1) Cyanophyceae
2) Chlorophyceae
3) Bacillariophyceae
4) Xanthophyceae.

The algae collected from all sampling stations were identified with the use of standard monographs Desikacharya (1959), Randhawa (1959), Pal et al. (1962), Ramanathan (1964), Bourrelly (1972), Tapale and Kamble (2014) Philipose (1967), Anand (1998), Tarar and 
Bodhe (1998a,1998b), Srinivasan (1969), Rajan (2000) Pal and Santra (1990), Tambe and Pingle (2014).

Tambe (2018), Tapale B.K. (2018) Identified algae have been enumerated systematically, monographs and available recent literature.

\section{RESULTS AND DISCUSSION:}

Total number of algal taxa observed during study period given in table-1. Dominance of Chlorophyceae was recorded at all the study sites. There are about 39 species of Chlorophyceaen algae.

Maximum number of algae was observed from December to April and to some extent in May. During and after rainy season benthic and filamentous algae were seen. The seasonal percentage of algae was found more in winter. More seasonal percentage of blue green in monsoon and Bacillariophyceae in summer. Formations of blooms of Microcystis, Oscillatoria, were also observed during summer. Diatoms were observed throughout the year and found by minimum in summer.

The total number of algal taxa recorded at three sampling stations is shown in Table no- 1

1) Cyanophyceae - In the present study Cyanophyceae (Blue green) were comparised to 17 species belonging to 11 genera and 3 orders as Chroococales, Oscillatoriales and Nostocales. The class was represented by most of the species of Chroococcus, Aphanocapsa, Merismopedia, Lyngbya, Anabaena, Nostoc, Gleocapsa, Scytonema, Dichothrix and Gloeotrichia.

2) Chlorophyceae (Green algae) - Green algae were dominant throughout investigation. Identified species of Ulothrix, Volvax, Chaetophora, Stigioclonium, Oedogonium, Closteridium, Pediastrum, Coelastrum, Oocystis Scenedesmus, Zygnema, Spirogyra, Closterium, Cosmarium, Chlorella, Ankistrodesmus, Tetraedron, Euastrum and Staurastrum, were observed during study period. There are about 33 species belongs to 24 genera of 11 families and 5 orders (Volvocales, Ulotricales, Chaetophorales, Oedogonials, and Zygnemetales.)

3) Bacillariophyceae - Bacillariophyceae was represented by 16 species, 7 genera, 3 families and 2 orders as Centrales and Pennales. Other diatoms comprises by species of Fragilaria, Navicula, Pinnularia, Cymbella, Cyclotella, and Gomphonema.

4) Xanthophyceae - Xanthophyceae represents only 2 genera belongs to Order Heterosiphonales and Family Vaucheriaceae

\section{Conclusion -}

Following points have emerged out from the present investigation. In the present study 81 algal taxa belonging to 53 genera, representing 24 families and 13 orders were recorded from three sites of Ambit dam Maximum number of algae was observed from December to April and some extent in May, from which Chlorophyceae were found dominant, during the period of investigation.

\section{REFERENCES:}

Anand N. (1998). Indian Fresh water micro algae. Published by Bishen Singh Mahendra Pal Singh, Dehradun PP-1-94

Bourrelly, P. (1972). Les algeus deau douce. Initiation a, la systematique. Tome I. Paris.E.D.N.Boubee, PP 1to 475

Desikachary, T.V. (1959). Cyanophyta ICAR, New Delhi.1-686.

Pal, B.P. Kundu B.C Sundarlingam, V.S. and G.J.Venkatraman. (1962). Charophyta, Indian Council of Agricultural Research, New Delhi. Gonzalves, E.A. 1981. Oedogonials. Indian Council of Agricultural Research. New Delhi. PP 1-557.

Pal. V.C.and Santra S.C. (1990). Algae of Midnapore, West Bengal. II Bacillariophyceae. Phykos 29(1\&2): 73-81 
Philipose, M.T. (1967). Chlorococcales. Indian Council of Agricultural Research, New Delhi. PP. 323

Rajan, S.S. (2000). Practical Manual of Algae.Anmol Publications Pvt.Ltd: New Delhi. PP.1-207

Randhawa M.S. (1959). Zygnemetaceae. Indian Council of Agricultural Research, New Delhi, pp. $1-478$

Ramanathan, K.R. (1964). Ulotricals. Indian Council of Agricultural Research, New Delhi, pp.I-ix,-1-188

Sinha, M. (1997). Limnological studies on a natural water body around Jaintpur Muzaaffarpur). Ph.D. Thesis, B.r.A. Bihar University.

Srinivasan, A. (1974) Limnological features of a tropical impoundment, Bhavanisagar reservoir (Tamil Nadu), India. Int. Rev Hydrobiol. 59(3):327-342.

Tambe,

D.B.and

Pingle,S.D.(2012)

Hydrobiological studies of Ambit
dam,Int.Advances in plant sciences 25(I) 6567

Tambe Deepmala (2018) Phytoplankton diversity of Bhandardara dam, Flora and Fauna international Journal 13 45-48

Tapale, B.K. and Kamble S.D. Ph.D Thesis Ecological studies of Nilwande dam (2014) Ph.D. thesis. BAMU, Aurangabad.

Tapale Balasaheb (2018) Biodiversity of Bhandardara reservoirs (Wilson Dam) of Akole Taluka. Scholarly Research journal for interdisciplinary studies 7/39 156-164

Tarar, J.L. and Bodkhe, Seema (1998a). Studies on Chlorococcales of Napery. Phykos 37(1 \& 2):107-114

Tarar, J.L. and Bodkhe, Seema (1998b). Studies on Diatoms Flora of polluted water bodies of Nagpure. Phykos 37 (1 \& 2): 77-86.

Trivedy, R.K. and Goel, P.K. (1986). Chemical and Biological Methods for Water Pollution Studies. Environmental Publications, Karad (India) pp.1-211.

Table No - 1. Enlisted Algae from Study Area

\begin{tabular}{|c|c|c|c|c|}
\hline \multirow{2}{*}{ Sr. No. } & \multirow{2}{*}{ Name of the alga } & \multicolumn{3}{|c|}{ Study stations } \\
\hline & & $\mathbf{S}_{1}$ & $\mathbf{S}_{2}$ & $\mathbf{S}_{3}$ \\
\hline & $\begin{array}{l}\text { Class- Cyanophyceae } \\
\text { Order- Chroococales } \\
\text { Family- Chroococaceae }\end{array}$ & & & \\
\hline 1. & Chroococcus minor (Kuctz) Naegeli & + & + & + \\
\hline 2. & Gleocapsa violacea (Corda) Rabenh & + & + & + \\
\hline 3. & Gleocapsa atrata (Turp.) Kuetz. & + & + & + \\
\hline 5. & Aphanothece pallida (Kuetz) Rabenh & + & + & + \\
\hline 7. & Merismopedia tenuissima Lemm & + & + & + \\
\hline \multirow[t]{2}{*}{8} & Microcystis protocystis (Crow) & + & + & + \\
\hline & $\begin{array}{l}\text { Order-Oscillatoriales } \\
\text { Family-Oscillatoriaceae }\end{array}$ & & & \\
\hline 9. & Spirulina laxisima West, G.S. & + & + & + \\
\hline 10. & Spirulina subsalsa Ornet. ex. Gomont & + & & + \\
\hline
\end{tabular}




\begin{tabular}{|c|c|c|c|c|}
\hline 11. & Oscillatoria subberevis Schmidle & + & + & + \\
\hline & $\begin{array}{l}\text { Order- Nostocales } \\
\text { Family-Nostocaceae }\end{array}$ & & & \\
\hline 12. & Anabaeno sphaerica Bornet et Flash & + & + & + \\
\hline \multirow[t]{2}{*}{13.} & Nostoc commune voucher ex Born et Flash & + & & + \\
\hline & Family- Scytonemataceae & & & \\
\hline 14. & Scytonema malviyaensis Bharadwaja & + & & + \\
\hline 15. & $\begin{array}{l}\text { Scytonema tolypothrichoides Kuetz.ex Born et } \\
\text { Flash }\end{array}$ & + & + & + \\
\hline \multirow[t]{2}{*}{16.} & Scytonema mirabile (Dillw.) Born. & & + & + \\
\hline & Family-Rivularaceae & + & & + \\
\hline \multirow[t]{3}{*}{17.} & Dichothrix gybsophila (Kuetz) Born.et.flash. & + & + & + \\
\hline & $\begin{array}{l}\text { Class-Chlorophyceae } \\
\text { Order-Volvocals }\end{array}$ & & & \\
\hline & Family-Volvocaceae & + & + & + \\
\hline 1. & Eudorina elegans Ehrenberg & + & + & + \\
\hline 2. & Pandorina cyclidricum Iyenger & + & + & + \\
\hline 3. & Volvox aureus Ehrenberg & + & + & + \\
\hline \multirow[t]{2}{*}{4.} & Volvox glabater Linn. & + & + & + \\
\hline & Family - Asterococcaceae & & & \\
\hline 5. & Chlamydocapsa ampla (Kuetz) Fott & + & + & + \\
\hline \multirow[t]{2}{*}{6.} & Chlamydocapsa plactonia (W.et.G.S.West) Fott & + & + & + \\
\hline & $\begin{array}{l}\text { Order-Ulotrichales } \\
\text { Family-Ulotrichaceae }\end{array}$ & & & \\
\hline 7. & Ulothrix zonata (weber\&mohr) Kuetz. & + & + & + \\
\hline \multirow[t]{2}{*}{8.} & Ulothrix cyclindricum Prescott & + & + & + \\
\hline & $\begin{array}{l}\text { Order -Chaetophorales } \\
\text { Family-Chaetophoraceae }\end{array}$ & & & \\
\hline 9. & Chaetophoa elegans (Roth.) C.A.Ag. & + & + & + \\
\hline \multirow[t]{2}{*}{10.} & Stigeoclonium tenue (C.A.Ag.)Kuetz & + & + & \\
\hline & $\begin{array}{l}\text { Order-Oedogonials } \\
\text { Family-Oedogoniaceae }\end{array}$ & & & \\
\hline 11. & Oedogonium latisculum Tiff & + & + & + \\
\hline 12. & Chlorococcum humicola Naegeli & + & + & + \\
\hline \multirow[t]{2}{*}{13.} & Closteridium cynthica De.Not & & + & + \\
\hline & Family- Hydrodictyaceae & & & \\
\hline 14. & Hydrodictyon reticulatum (L) Lagerbeim & + & + & + \\
\hline 15. & Pediastrum simplex vor. Duodenarium (Bailey) & + & + & + \\
\hline 16. & Pediastrum ovatum (Her.) A.Braun & & + & + \\
\hline
\end{tabular}




\begin{tabular}{|c|c|c|c|c|}
\hline 17. & Pediatrum duplex subgranulatum Raciborski & + & + & \\
\hline \multirow[t]{2}{*}{18.} & Pediatrum boryanum (Turp) Meneghini & + & + & + \\
\hline & Family - Coelastraceae & & & \\
\hline \multirow[t]{2}{*}{19.} & Coelastrum reticulatum (Dang) sen & + & + & + \\
\hline & Family-Oocystaceae & & & \\
\hline 20. & Tetraedron regulare (kuetzing) & + & + & + \\
\hline 21. & Oocystis elliptica W.West & + & + & + \\
\hline 22. & Chlorella ellipsoda & & + & + \\
\hline \multirow[t]{2}{*}{23.} & Tetrahedron gracile (Reinch) Hansgirg & + & + & + \\
\hline & Family-Scenedesmaceae & & & \\
\hline 24. & $\begin{array}{l}\text { Scenedesmus bijugatus var. alternanas } \\
\text { (reinsch)Hansgirg }\end{array}$ & + & + & \\
\hline \multirow[t]{2}{*}{25.} & Scenedesmus dimorphus (Turpin) Kuetzing & & + & + \\
\hline & $\begin{array}{l}\text { Order- Zygnematales } \\
\text { Family-Zygnemataceae }\end{array}$ & & & \\
\hline 26. & Spirogyra setiformis (Roth) & + & + & + \\
\hline 27. & Spirogyra condensate (voucher)Kuetzing & + & + & + \\
\hline 28. & Zygnema pectinatum (Vauch) C.A Agardh. & + & + & + \\
\hline \multirow[t]{2}{*}{39.} & Zygnema extenue Jao. & + & + & + \\
\hline & Family-Desmidiaceae & & & \\
\hline 30. & Closterium acutum (Lyngb) Breb & + & + & + \\
\hline 31. & Euastrum subintegrum Norsted. & + & + & + \\
\hline 32. & Staurastrum leptocladwm Norsted. & & + & + \\
\hline \multirow[t]{2}{*}{33.} & Cosmarium awadhense Prasad \& Mehrotra & + & + & + \\
\hline & $\begin{array}{l}\text { Class-Xanthophyceae } \\
\text { Order-Heterosiphonales } \\
\text { Family-Vaucheriaceae }\end{array}$ & & & \\
\hline 1. & Vaucheria terrestris Lyngbye em.Walz & + & + & + \\
\hline \multirow[t]{2}{*}{2.} & Vaucheria pachyderma Walz. & + & + & \\
\hline & $\begin{array}{l}\text { Class-Bacillariophyceae } \\
\text { Order-Centrales } \\
\text { Family-Coscinodiscaceae }\end{array}$ & & & \\
\hline 1. & Melosira granulata (Ehr) Ralf. & + & + & + \\
\hline 2. & Melosira juergensii Agardh & + & + & + \\
\hline \multirow[t]{2}{*}{3.} & Cyclotella stelligera Cleve et.Grum & + & + & + \\
\hline & $\begin{array}{l}\text { Order-Pennales } \\
\text { Family-Naviculaceae }\end{array}$ & & & \\
\hline 4. & Cymbella offinis Kuetz & + & + & + \\
\hline 5. & Cymbella bharatensis (Sarode and Kamat) & + & + & + \\
\hline 6. & Cymbella chandolensis (Gandhi) & + & + & + \\
\hline
\end{tabular}


I J R B A T, Issue (VIII), Vol. II, May 2020: 125-130

\begin{tabular}{|c|c|c|c|c|}
\hline 7. & Cymbella cymbeliformis (Kuetz) & + & + & + \\
\hline 8. & Gomphonema tingulatum Ehr. & + & + & + \\
\hline 9. & Gomphonema vidharbhensis (Sarode and Kamat) & & + & + \\
\hline 10. & Gomphonema clavatoides Gandhi & + & + & \\
\hline 11. & Navicula grimii Krasske & + & + & + \\
\hline 12. & Navicula sabyadrensis Sarode and Kamat & + & + & + \\
\hline 13. & Pinnularia acrosphaeria (Breb) W.Smith & + & + & + \\
\hline \multirow[t]{2}{*}{14.} & Pinnularia palea (Kuetz) W.Smith & + & + & + \\
\hline & Family - Fragilariaceae & + & + & + \\
\hline 15. & Fragilaria brevistriata Grun & + & + & + \\
\hline 16. & Fragilaria virescens var. Capitata. Krasske & + & + & + \\
\hline
\end{tabular}

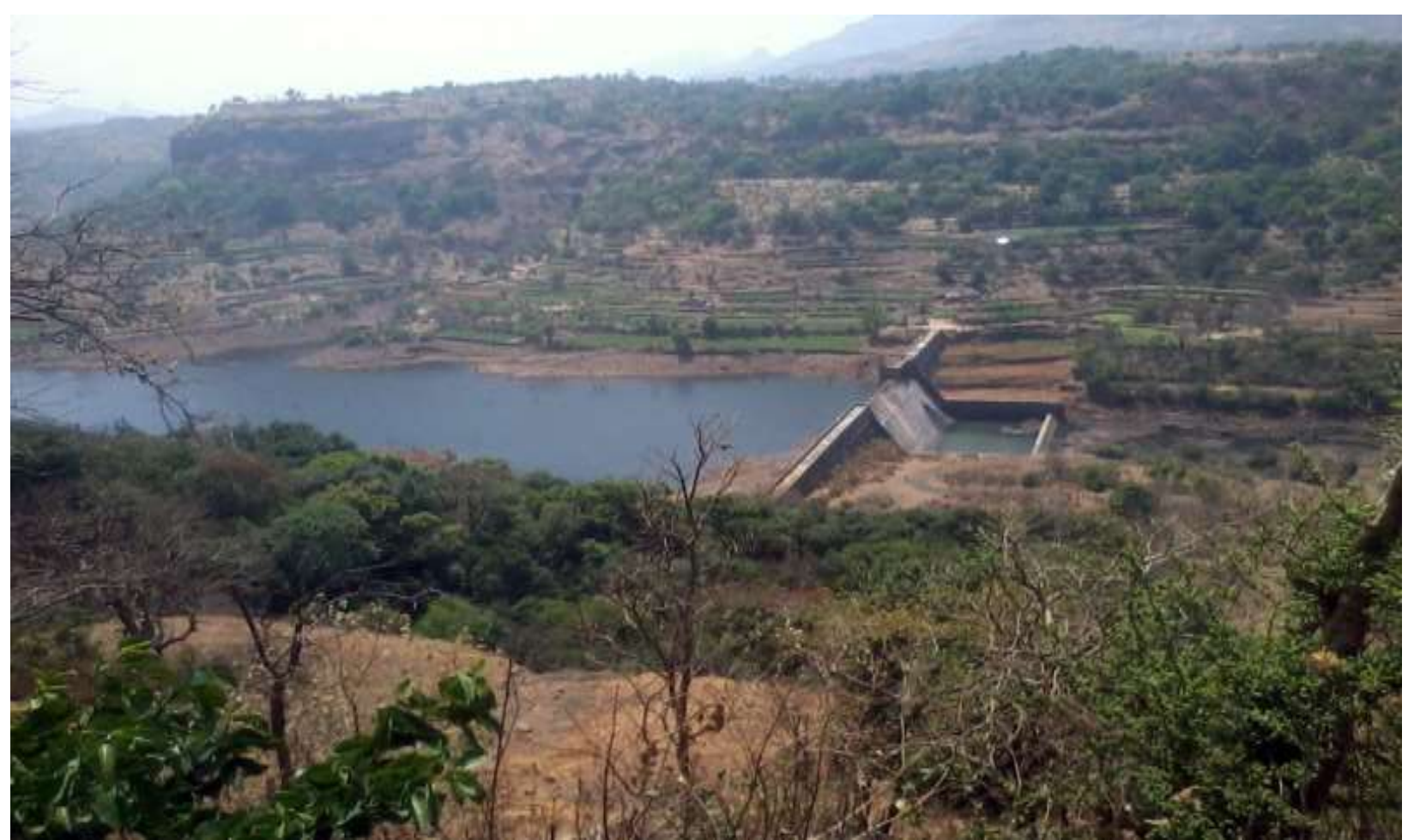

Figure 1. Showing Ambit Dam and its water. 